A modal actuator-based single-layer piezoelectric transformer for coilless soft switching inverters

This article has been downloaded from IOPscience. Please scroll down to see the full text article. 2007 Smart Mater. Struct. 162046

(http://iopscience.iop.org/0964-1726/16/6/007)

The Table of Contents and more related content is available

Download details:

IP Address: 140.112.113.225

The article was downloaded on 25/12/2008 at 03:26

Please note that terms and conditions apply. 


\title{
A modal actuator-based single-layer piezoelectric transformer for coilless soft switching inverters
}

\author{
Yao Tien Huang ${ }^{1}$, Wen Jong $\mathrm{Wu}^{2}$, Yu Yuan $\mathrm{Chen}^{1}$ and \\ Chih Kung Lee L $^{1,2,3}$ \\ ${ }^{1}$ Institute of Applied Mechanics, National Taiwan University, Taipei, 10617 Taiwan \\ ${ }^{2}$ Department of Engineering Science and Ocean Engineering, National Taiwan University, \\ Taipei, 10617 Taiwan \\ E-mail: cklee@ntu.edu.tw
}

Received 21 August 2006, in final form 2 August 2007

Published 12 September 2007

Online at stacks.iop.org/SMS/16/2046

\begin{abstract}
In this paper, we propose a coilless backlight inverter for LCD TVs. To simplify the manufacturing procedures of the inverter, a single-layer piezoelectric transformer was used as the basis for the development. Traditionally, the input capacitance of a single-layer piezoelectric transformer prevents us from identifying a proper inductor to achieve $L C$ resonance. Without the presence of $L C$ resonance, high-frequency noise within the output signal can be found. The zero-voltage switching technique can be used to adjust the rectified input voltage to become a trapezoidal voltage waveform and thus reduce some of the high-frequency voltage signals amplified from the input voltage waveform. As the presence of this high-frequency voltage waveform will influence the vibration of the Rosen-type piezoelectric transformer, which can lead to a lower energy transfer efficiency and higher temperature rise on the MOSFET, a solution is needed to further enhance the performance of the piezoelectric transformer-based inverter. It can be shown that the above problems can be solved using a quasi-modal surface electrode adopted onto the surface of a piezoelectric transformer. More specifically, modal filtering provided by the surface electrode of the piezoelectric transformer can be shown to facilitate the removal of the inductor and to eliminate the high-frequency noise that cannot be eliminated by a soft switching technique. In addition, the temperature rise of the MOSFET within the driving circuitry can be shown to be improved significantly. The experimental results were found to match well with the theoretical predictions.
\end{abstract}

\section{Introduction}

The piezoelectric transformer was first invented by Rosen [1] in 1957 using mechanical vibration instead of magnetic flux to transform electrical energy. As a mechanical structure usually has a higher quality factor than that of electromagnetic components, a piezoelectric transformer can potentially possess a much higher energy transfer efficiency than an

3 Address for correspondence: Institute of Applied Mechanics, National Taiwan University, No. 1, Sec. 4, Roosevelt Road, Taipei, 10617 Taiwan. electromagnetic transformer. A piezoelectric transformer possesses many advantages, such as high energy transfer efficiency, low electromagnetic interference, small volume and low temperature rise. Although all these advantages have attracted much research in this field, the most noteworthy application is in the key component which powers the cold cathode fluorescent lamp (CCFL) used in the backlight of liquid crystal displays (LCDs). More specifically, the automatic adjustment of the piezoelectric transformer stepup ratio with respect to the loading impedance makes the 
piezoelectric transformer a natural choice to light plasmabased devices such as CCFLs.

A multi-layer piezoelectric transformer was the main research direction for CCFL applications in the 1990s when small LCD displays required a high step-up ratio and a proper input impedance match [2]. One of the main achievements during that time was the placement of an inductance in front of the multi-layer piezoelectric transformer in order to arrive at an $L C$ resonance condition. This condition was met when the resonance frequency formed by the inductance $L$ and the static input capacitance $C$ of the piezoelectric transformer matched that of the operating resonance frequency of the piezoelectric transformer. Even though a multilayer piezoelectric transformer offers the high step-up ratio needed and the $L C$ resonance condition provides us with an opportunity to filter out the high frequency noise present within the input voltage, the complicated manufacturing process made it difficult to compete with traditional coil transformers.

The soft switching technique, a technique that is important for a switched-mode power supply (SMPS), was first adopted to develop a disc-type piezoelectric transformer-based inverter for lighting a $40 \mathrm{~W}$ fluorescent lamp [3]. One fundamental difference between the disc-type piezoelectric transformer and the Rosen-type piezoelectric transformer is in the location of the higher harmonics. More specifically, the higher harmonics of a Rosen-type piezoelectric transformer sits at integer multiples of the fundamental frequency. This situation can be automatically circumvented by the disc-type piezoelectric transformer as the resonant frequencies of its higher harmonics are not integer multiples of the fundamental frequency. More specifically, considering the Fourier components of a square wave or a trapezoidal wave, its higher harmonics frequency are odd integer multiples of the fundamental mode frequency, i.e. 3, 5.7, etc., times the fundamental frequency. All of which basically state that injecting a square or trapezoidal input waveform into a disc-type piezoelectric transformer will not excite higher harmonics of the modes of interest. This is the underlying reason that a soft switching technology can be used to develop a coilless disc-type piezoelectric inverter [4-6].

For LCD TV backlights, since the impedance of the CCFL is much higher than that of fluorescent lamps (FL), a Rosentype piezoelectric transformer remains the only choice when trying to match a CCFL impedance. As the higher harmonics of a Rosen-type piezoelectric transformer overlaps with the high frequency component inside a trapezoidal input voltage, the soft switching technology cannot be directly applied to the driving circuit of the piezoelectric inverter for CCFLs. It is due to this reason that, even though a soft switching technology was used in 2002 to drive a Rosen-type piezoelectric transformer, both the input power and the efficiency obtained were low [7].

The modal actuator and sensor were invented by Lee in 1989 [8-10]. Extending that line of concept, Lee et al developed a quasi-modal piezoelectric transformer in 2003 [11, 12]. Traditionally, modal sensors and actuators are developed by having the surface electrode designed according to the distribution of the stress field of the structure of interest. In developing the quasi-modal piezoelectric transformer, the input section of the piezoelectric transformer was designed to have the surface electrode match the stress field as much as possible while minimizing the potential to excite the higher harmonics of the transformer. The experimental results show that adopting a quasi-modal input electrode can improve the energy transfer efficiency and reduce the temperature rise. In 2005, a single-layer piezoelectric transformer with a quasimodal electrode was used to light a $650 \mathrm{~mm}$ long CCFL successfully [13]. Although the quasi-modal electrode has a filtering function, it still cannot achieve the real coilless piezoelectric-based inverter. The underlying reason is that the piezoelectric transformer may act as a capacitor. If the switching stage sees a capacitance load, switching loss will be high and inefficient switch working operations will be induced. To solve the problem of switching loss, the soft-switching technique should be taken into consideration.

The underlying innovation of this paper is based on the integration of the quasi-modal electrode and a soft switching technique. By using a soft switching technology, the rectified input voltage can be adjusted to become trapezoidal in shape. By adopting a quasi-modal electrode at the input section of the Rosen-type piezoelectric transformer which acts as a perfect band-pass filter, the residual higher harmonics of the input voltage waveform will not excite the higher harmonics of the piezoelectric transformer fundamental modes. Rosen-type piezoelectric transformers of two different thicknesses were manufactured to verify the effect of combining the two abovementioned technologies. With proof of the advantages of the integration, a coilless single-layer piezoelectric transformerbased inverter was completed which can adopt a soft switching technology to improve the total energy transfer efficiency. This single-layer piezoelectric transformer, which can be manufactured in a simple manufacturing process, will be suitable for mass production. It should be noted that the adoption of a soft switching technology provides us with an opportunity to replace the magnetic component in front of the piezoelectric transformer, which essentially removes the problem of requiring an unreasonable large inductance to match the small input capacitance of a single-layer piezoelectric transformer for LCD TV backlights. In addition, this newly developed piezoelectric transformer-based inverter directly utilizes line voltage as its input in order to reduce the amplification needed and which eliminates the need for using a multi-layer piezoelectric transformer.

\section{Design of a modal electrode for a Rosen-type piezoelectric transformer}

The configuration of the single-layer piezoelectric transformer with a quasi-modal input electrode is shown in figure 1 . The piezoelectric transformer performs voltage amplifications by using the second resonant mode. The appearance looks like a regular Rosen-type piezoelectric transformer except that the input electrode was designed to match the modal stress distribution of the piezoelectric transformer. The effect of the full modal filtering effect is analyzed below.

The governing equation of the piezoelectric transformer is represented as [11-14]

$$
c_{11}^{E} \frac{\partial^{2} u(x, t)}{\partial x^{2}}-\rho \frac{\partial^{2} u(x, t)}{\partial t^{2}}=E_{3}^{\text {in }} e_{31} \frac{\partial S(x)}{\partial x},
$$

where $u(x, t)$ represents the displacement along the $x$ direction (1 direction), $E_{3}^{\text {in }}$ represents the input electric field along 


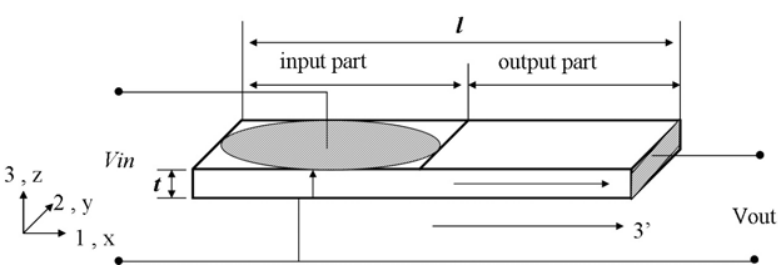

Figure 1. Configuration of a Rosen-type piezoelectric transformer with a quasi-modal electrode.

the $z$ direction (3 direction), $S(x)$ represents the shape of the input electrode, $e_{31}$ is the piezoelectric stress/electric field constant, $\rho$ is the density of the piezoelectric material and $c_{11}^{E}$ represents the elastic constant along the $x$ direction (1 direction). Following a traditional piezoelectric sign convention, the poling direction of the output section will be denoted as the $3^{\prime}$ axis since typically the $3^{\prime}$ axis denotes the poling direction and the " " is used to represent a local axis that can be integrated into the global coordinates, i.e. the 1 , 2,3 axes. That is, the electric output of the piezoelectric transformer will be extracted from the axis, i.e. $D_{3^{\prime}}^{\text {out }}$ represents the output electric displacement. The boundary conditions at the two ends $x=0$ and $l$ of the piezoelectric transformer are thus

$$
\begin{gathered}
\left.\frac{\partial u}{\partial x}\right|_{x=0}=\left.\frac{e_{31} E_{3}^{\mathrm{in}}(t)}{c_{11}^{E}}\right|_{x=0}, \quad \text { and } \\
\left.\frac{\partial u}{\partial x}\right|_{x=l}=\frac{h_{3^{\prime} 3^{\prime}} D_{3^{\prime}}^{\text {out }}(l, t)}{c_{3^{\prime} 3^{\prime}}^{D}},
\end{gathered}
$$

where $c_{3^{\prime} 3^{\prime}}^{D}$ is the elastic constant along the $3^{\prime}$ direction, and $h_{3^{\prime} 3^{\prime}}$ is the piezoelectric stress/electric displacement constant. By using an eigenfunction expansion, the displacement field $u(x, t)$ and the corresponding boundary conditions can be represented as

$$
\begin{gathered}
u(x, t)=\sum_{i=1}^{\infty} A_{i}(t) \varphi_{i}(x), \\
\frac{\mathrm{d}^{2} \varphi_{i}}{\mathrm{~d} x^{2}}+\lambda_{i} \varphi_{i}=0, \quad \frac{\mathrm{d} \varphi_{i}}{\mathrm{~d} x}(0)=0 \quad \frac{\mathrm{d} \varphi_{i}}{\mathrm{~d} x}(l)=0
\end{gathered}
$$

where $\varphi_{i}(x)$ represents the $i$ th mode shape of the piezoelectric transformer and $A_{i}(t)$ represents the specific weighting factor along the $i$ th modal coordinates. Substituting equations (3) and (4) into equation (1), the governing equation can be transferred from a partial differential equation into a set of ordinary differential equations such as

$$
\begin{aligned}
& \frac{\mathrm{d}^{2} A_{i}(t)}{\mathrm{d} t^{2}}+\frac{c_{11}^{E}}{\rho} \lambda_{i} A_{i}(t)=q_{i}(t)+\frac{c_{11}^{E}}{\rho} \sqrt{\frac{2}{l}}\left[(-1)^{i+1}\right. \\
& \left.\times \frac{h_{3^{\prime} 3^{\prime}} D_{3^{\prime}}^{\text {out }}(l, t)}{c_{3^{\prime} 3^{\prime}}^{D}}-\left.\frac{e_{31} E_{3}^{\mathrm{in}}(t)}{c_{11}^{E}}\right|_{x=0}\right], \quad \text { and } \\
& q_{i}(t)=\int_{0}^{l}\left(-\frac{e_{31} E_{3}^{\mathrm{in}}(t)}{\rho} \frac{\partial S(x)}{\partial x}\right) \varphi_{i} \mathrm{~d} x ; \\
& \varphi_{i}(x)=\sqrt{\frac{2}{l}} \cos \left(\frac{\mathrm{i} \pi x}{l}\right),
\end{aligned}
$$

where $q_{i}(t)$ is the distributed forcing term induced by the input electrical field, $E_{3}^{\text {in }}$ and the last two terms from the boundary conditions. In order to simplify the influence of the boundary condition, the piezoelectric transformer can be assumed to operate under an open output condition. The output current of the piezoelectric transformer under an open condition is zero, and thus the output electric displacement $D_{3^{\prime}}^{\text {out }}(l, t)$ is also zero. It should be noted that a piezoelectric transformer typically operates with a loading impedance connected to the output section. From equation (5) it is clear that the boundary conditions will influence the vibration of the piezoelectric transformer. In addition, the displacement of piezoelectric material would also influence the output electric displacement $D_{3^{\prime}}^{\text {out }}(l, t)$. This strong coupling basically states that it is difficult to get a clear solution by trying to solve the governing equation directly. To circumvent this difficulty, the vibration behavior of the piezoelectric transformer was analyzed by assuming an open output condition as stated above. For the case when the loading impedance is connected to the piezoelectric transformer, the electromechanical interactions can be analyzed by adopting an equivalent circuit modal as traditionally done. More specifically, the equivalent circuit model was not adopted in this paper until after the normal mode expansion (equation (3)) was used to expand the governing equation (equation (1)). As the input electric field acts as a distributed force exerting through the surface electrode to the piezoelectric transformer, the last term of equation (5) on the right-hand side will be zero in the boundary. This is the same result as the theoretical analysis which was completed in the past $[13,14]$, where a driving factor $r_{i}$ and a normalized driving factor $r_{i b}$ were used to verify the modal filtering effect. We can see that

$$
r_{i}=-\frac{e_{31} E_{3}^{\mathrm{in}}}{\rho} \sqrt{\frac{2}{l}}\left[\int_{0}^{l} \frac{\partial S(x)}{\partial x} \cos \left(\frac{\mathrm{i} \pi x}{l}\right) \mathrm{d} x\right],
$$

and

$$
r_{i b}=-\frac{1}{i^{2}} \frac{r_{i}}{\frac{e_{31}\left|E_{3}^{\text {in }}\right|}{\rho} \sqrt{\frac{2}{l}}},
$$

where $r_{i}$ is the driving factor which represents the amplitude of the forcing term. More specifically, $r_{i}$ drives the vibration of the transformer and is associated with the input electric field. In this paper, the driving factor can be used to verify the effect of the modal electrode. If higher vibration modes are filtered out by the modal electrode, the associated driving factors will be zero, i.e. no vibration will be excited at these modes. In order to further simulate the nature of higher attenuation on a higher vibration mode, the normalized driving factor $r_{i b}$ can be obtained by normalizing $r_{i}$ with $i^{2}$. We can then compare the normalized driving factor on different modes with an attempt to verify the modal filtering effect on the piezoelectric transformers equipped with a quasi-modal and an uniform electrode. The shape of input electrode $S(x)$ for the quasi-modal and the uniform electrode-based piezoelectric transformers are

$$
\begin{gathered}
S(x)_{\text {quasi-modal }}=\sin \left(\frac{2 \pi x}{l}\right)\{H[x]-H[x-a l]\} ; \\
S(x)_{\text {uniform }}=H[x]-H[x-a l] .
\end{gathered}
$$

The simulated results of the driving factor $r_{i b}$ for the case where the length of the input electrode is equal to $l / 2$ is shown 
A modal actuator-based single-layer piezoelectric transformer for coilless soft switching inverters

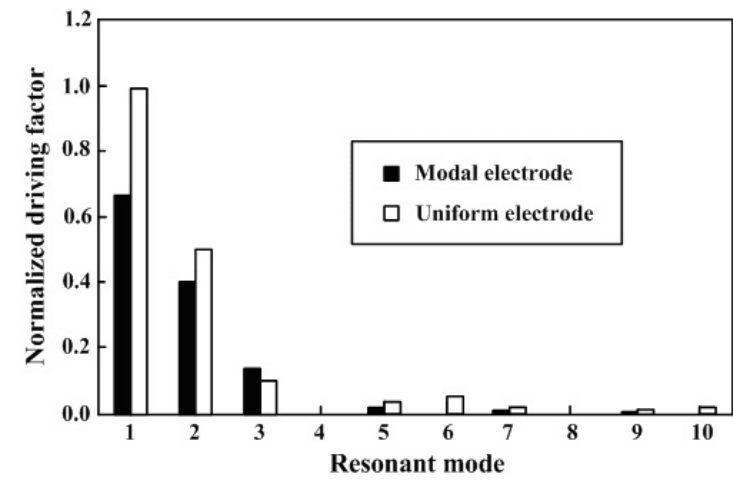

Figure 2. Normalized driving factor for different vibration modes of a modal and a uniform surface electrode-based piezoelectric transformer.

in figure 2. Since the piezoelectric transformer operates at the second resonant mode and it is clear from figure 2 that the sixth and the tenth driving factors of the piezoelectric transformer with a quasi-modal electrode is zero, this theoretical result basically states that the quasi-modal electrode can provide a full modal filtering effect for a rectified input voltage. For a trapezoidal input voltage, the modal electrode can also provide a full modal filtering effect. That is to say, the piezoelectric transformer with a quasi-modal electrode can be viewed as a perfect band-pass filter and the magnetic component free soft switching driving circuit can thus be used to directly drive it.

In order to verify the theoretical results mentioned above, Rosen-type piezoelectric transformers with two different electrodes were fabricated. In total, four different types of piezoelectric transformers were fabricated, which were denoted as PTU2MM, PTM2MM, PTM1MM and PTU1MM. PTU2MM was a $2 \mathrm{~mm}$ thick piezoelectric transformer with an uniform electrode while PTM2MM was a $2 \mathrm{~mm}$ thick piezoelectric transformer with a quasi-modal electrode. PTM1MM was a $1 \mathrm{~mm}$ thick piezoelectric transformer with a quasi-modal electrode and PTU1MM was a $1 \mathrm{~mm}$ thick piezoelectric transformer with an uniform electrode. All the piezoelectric transformers were fabricated by Eleceram Technology Co. Ltd, Taiwan.

To verify the modal filtering effects, an impedance analyzer, Agilent 4294, was used to measure the input impedance of all four piezoelectric transformers. The impedances of the four piezoelectric transformers are shown in figures 3 and 4 . In figure 3, it can be seen that the two different types of piezoelectric transformers (PTU2MM and PTM2MM) have resonant and anti-resonant peaks at the second resonant frequency. However, at the sixth and tenth resonant frequencies, the input impedance of PTM2MM possesses the characteristics of a static capacitance. These experimental results clearly indicate that a quasi-modal electrode can provide a full modal filtering effect for a rectified input voltage. Figure 4 shows that PTM1MM also possesses a modal filtering effect when compared to PTU1MM, which does not.

To verify the effects of a modal filtering electrode, an interferometer with nanometer resolution and $20 \mathrm{MHz}$ bandwidth [15] manufactured by Taiwan's Sunwave Optoelectronics, Inc. was used to measure the vibration of PTU1MM and
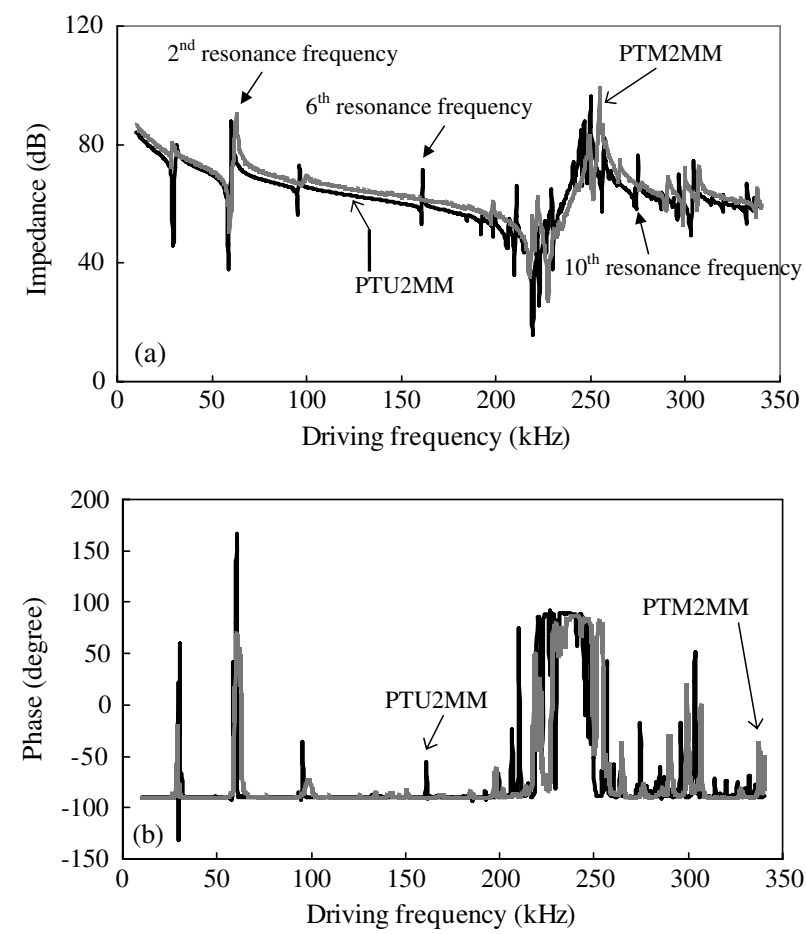

Figure 3. Magnitude (a) and phase (b) of the input impedance of a PTU2MM and a PTM2MM.
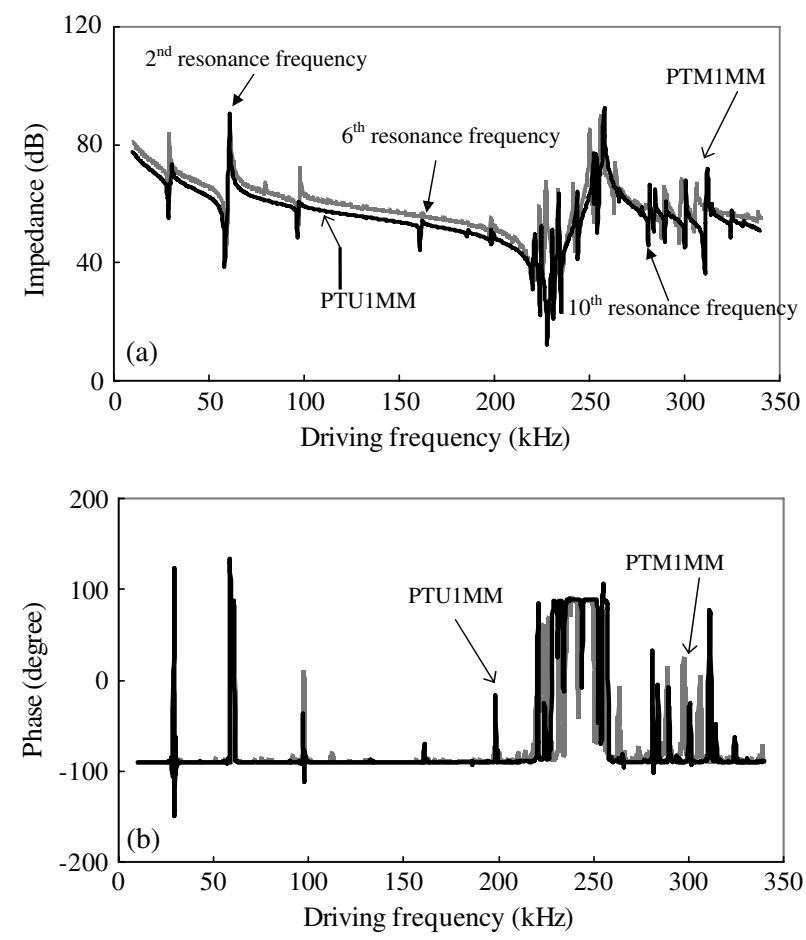

Figure 4. Magnitude (a) and phase (b) of the input impedance of a PTU1MM and a PTM1MM.

PTM1MM. The driving frequency varied from 40 to $200 \mathrm{kHz}$ while the lateral side surface displacement of the piezoelectric transformer was measured by the interferometer. The experimental results obtained when the amplitude of the input 


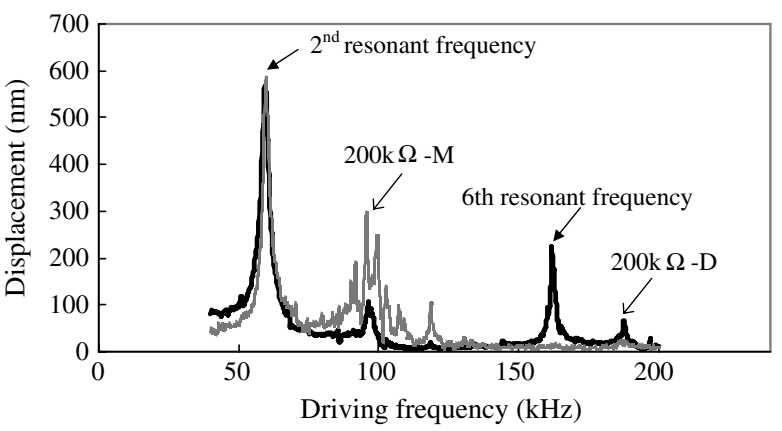

Figure 5. Displacement of a PTU1MM and PTM1MM at different driving frequencies when connected to a $200 \mathrm{k} \Omega$ load.

voltage were kept constant are shown in figure 5. The data line $200 \mathrm{k} \Omega$-D was obtained by connecting PTU1MM to a $200 \mathrm{k} \Omega$ load. Similarly, the data line $200 \mathrm{k} \Omega-\mathrm{M}$ was measured by connecting PTM1MM to a $200 \mathrm{k} \Omega$ load. At the second resonant frequency, both piezoelectric transformers were found to vibrate which is the desirable vibration mode. However, only PTU1MM and not PTM1MM vibrated at the sixth resonant frequency. From the experimental results shown, it is clear that a modal electrode can provide a full modal filtering effect for a rectified input voltage. In summary, by using a quasimodal electrode, a piezoelectric transformer would not transfer energy at the sixth resonant frequency and thus the electrical property of the piezoelectric transformer can be seen as possessing characteristics similar to a static capacitance.

\section{Soft switching technology}

A soft switching technology was used to design the driving circuit of a piezoelectric transformer over a long period of time [3-6]. The main design thinking comes from considering a traditional electronic ballast circuit which uses an $L-C$ resonant tank to generate an extra-high voltage for igniting a lamp. A traditional ballast circuit contains a series resonant inductor $L$, a series resonant capacitor $C$, a voltage stepup transformer and a high voltage resonant capacitor $C_{d 2}$ (figure 6). In the past, an equivalent circuit was used to simulate a piezoelectric transformer [16, 17]. For the case where the piezoelectric transformer works near a resonant frequency, an ordinary differential equation can be used to simulate it. Each ordinary differential equation can then be replaced by using an equivalent circuit model (figure 7). In figures 6 and 7, we can see that the piezoelectric transformer can be used to replace a traditional ballast circuit for CCFL lighting and where the design can be in figure 8 . The internal inductance $L$ of the ballast circuit can help reach the zerovoltage switching (ZVS) condition, which forms the basis of designing the driving circuit for the piezoelectric transformer. The main design thinking of the ZVS technology is to use the internal inductance $L$ of the piezoelectric transformer to charge/discharge the input capacitance $C_{d 1}$ and the parasitical capacitance of MOSFET. The soft switching process follows the following steps. The MOSFET switch $S_{2}$ turns off first. In the meantime, switch $S_{1}$ does not turn on immediately as the current from the inductor will charge the input capacitance $C_{d 1}$ and raise the voltage $V_{C_{d 1}}$. When the voltage $V_{C_{d 1}}$ increases

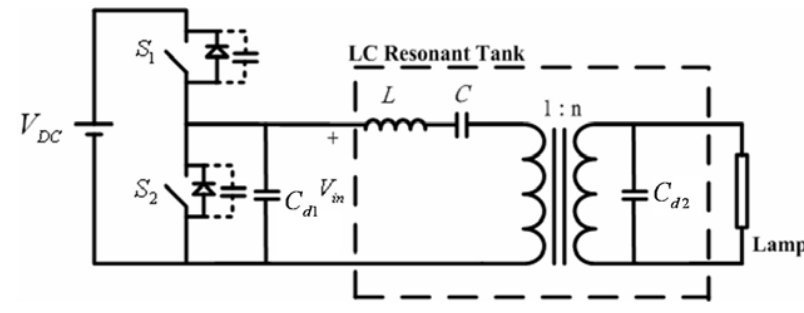

Figure 6. Configuration of a traditional electronic ballast circuit.

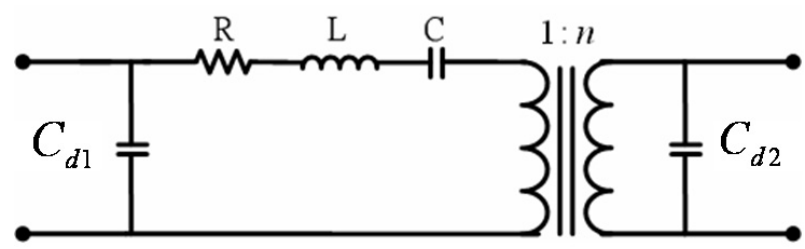

Figure 7. An equivalent circuit of a piezoelectric transformer

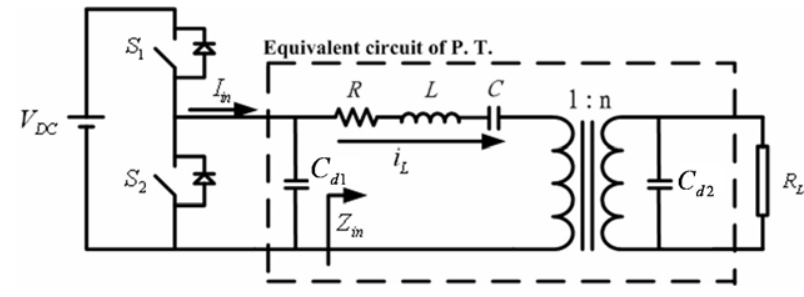

Figure 8. Configuration of a piezoelectric transformer-based ballast circuit.

to $V_{\mathrm{DC}}$, the internal diode of switch $S_{1}$ is then turned on. Then voltage $V_{C_{d 1}}$ stops increasing and remains equal to voltage $V_{\mathrm{DC}}$. If switch $S_{1}$ is turned on at this time, the switch is then called to switch under the ZVS condition. For the second part of the switching process, the MOSFET switch $S_{1}$ is turned off. In the meantime, the switch $S_{2}$ does not turn on immediately and the current of the inductance starts to discharge the input capacitance $C_{d 1}$ such that the voltage $V_{C_{d 1}}$ starts to decrease. As soon as the voltage $V_{C_{d 1}}$ decreases to zero, the internal diode of the switch $S_{2}$ is turned on. The voltage $V_{C_{d 1}}$ then stops decreasing and is then equal to the electric ground. If switch $S_{2}$ is then turned on at this time, the switch is again called to switch under the ZVS condition.

The waveform of the input voltage $V_{C_{d 1}}$ can be adjusted from a rectified type to a trapezoidal shape by using the abovementioned soft switching technology. In order to verify the ZVS condition, theoretical analysis can be used to predict the charge/discharge voltage. The inductance $L$ is used to charge/discharge the capacitance $C_{d 1}$ and can be written as [3]

$$
\mathrm{d} Q=C_{d 1} \mathrm{~d} V=i_{L} \mathrm{~d} t,
$$

where $Q, i$ and $V$ represent the charge, current and voltage, respectively. In order to achieve the ZVS condition, the voltage difference of the input capacitor $C_{d 1}$ must be larger than $V_{\mathrm{DC}}$ during the dead time. The mathematic relationship can be represented as

$$
V_{C_{d 1}, \mathrm{pk}}=\frac{Q}{C_{d 1}} \geqslant V_{\mathrm{DC}}
$$


Table 1. Parameters of a piezoelectric transformer equivalent circuit

\begin{tabular}{lllrrrr}
\hline $\begin{array}{l}\text { Thickness } \\
(\mathrm{mm})\end{array}$ & $R(\Omega)$ & $L(\mathrm{~F})$ & $C(\mathrm{pF})$ & $C_{d 1}(\mathrm{pF})$ & $C_{d 2}(\mathrm{pF})$ & $n$ \\
\hline 2 & 46.010 & 0.1205 & 59.393 & 628.559 & 6.696 & 7.36 \\
1 & 16.870 & 0.0653 & 110.116 & 1387.310 & 2.883 & 15.22 \\
\hline
\end{tabular}

where $V_{C_{d 1} \text {,pk }}$ represents the peak value of the voltage across the input capacitance $C_{d 1}$. Excluding the input capacitance $C_{d 1}$, the input impedance of the piezoelectric transformer can be written as

$$
Z_{\text {in }}(f)=R_{\text {in }}(f)+\mathrm{j} X_{\text {in }}(f),
$$

where $f$ is the driving frequency of the input voltage, and $R_{\text {in }}$ and $X_{\text {in }}$ are the real part and the imaginary part of the input impedance, respectively. The fundamental component of the trapezoidal input voltage can then be written as

$$
V_{\text {in }}(t)=V_{\mathrm{DC}} \frac{2}{\pi} \frac{\sin \left(\pi t_{r} f\right)}{\pi t_{r} f} \sin (2 \pi f t),
$$

where only the fundamental component of the input voltage that charges/discharges $C_{d 1}$ is considered. By using equations (10), (12) and (13), the charged voltage across the input capacitance can be represented as

$$
\begin{aligned}
V_{C_{d 1}}(f)=\frac{V_{\mathrm{DC}} \frac{2}{\pi} \frac{\sin \left(\pi t_{r} f\right)}{\pi t_{r} f}}{C_{d 1} 2 \pi f} \\
\times \frac{R_{\text {in }}(f)^{2}-X_{\text {in }}(f)^{2}-R_{\text {in }}(f) \sqrt{X_{\text {in }}(f)^{2}+R_{\text {in }}(f)^{2}}}{\left(X_{\text {in }}(f)^{2}+R_{\text {in }}(f)^{2}\right) \sqrt{X_{\text {in }}(f)^{2}+R_{\text {in }}(f)^{2}}} .
\end{aligned}
$$

In equation (14), we can see that the charged voltage $V_{C_{d 1}}$ is related to the input impedance $Z_{\text {in }}(f)$, input capacitance $C_{d 1}$ and the dead time $t_{r}$. This equation can be used to calculate the magnitude of the charged voltage $V_{C_{d 1}}$ for different working frequencies. We can also derive the optimal working frequency region where the MOSFET can operate under a ZVS condition within this region.

In order to verify the operation of the soft switching technology, an equivalent circuit of PTM1MM and PTM2MM was measured by using an Agilent 4294 Impedance Analyzer. The measured value of the equivalent circuit is shown in table 1. It is clear from table 1 that, if the thickness of the piezoelectric transformer changes from 2 to $1 \mathrm{~mm}$, the input capacitance $C_{d 1}$ will double. Similarly, the step-up ratio of the internal ideal transformer also increases two times. Considering the case when the input voltage remains constant, the current through the internal inductance $L$ will then increase by four times.

From equation (14), the value of charged voltage $V_{C_{d 1}}$ can be used to determine whether the switch can lead us to obtain a ZVS condition or not. To verify the ZVS condition clearly, equation (14) can be rewritten as

$$
\begin{aligned}
& \frac{V_{C_{d 1}}(f)}{V_{\mathrm{DC}}}=\frac{\frac{2}{\pi} \frac{\sin \left(\pi t_{r} f\right)}{\pi t_{r} f}}{C_{d 1} 2 \pi f} \\
& \quad \times \frac{R_{\text {in }}(f)^{2}-X_{\text {in }}(f)^{2}-R_{\text {in }}(f) \sqrt{X_{\text {in }}(f)^{2}+R_{\text {in }}(f)^{2}}}{\left(X_{\text {in }}(f)^{2}+R_{\text {in }}(f)^{2}\right) \sqrt{X_{\text {in }}(f)^{2}+R_{\text {in }}(f)^{2}}} .
\end{aligned}
$$
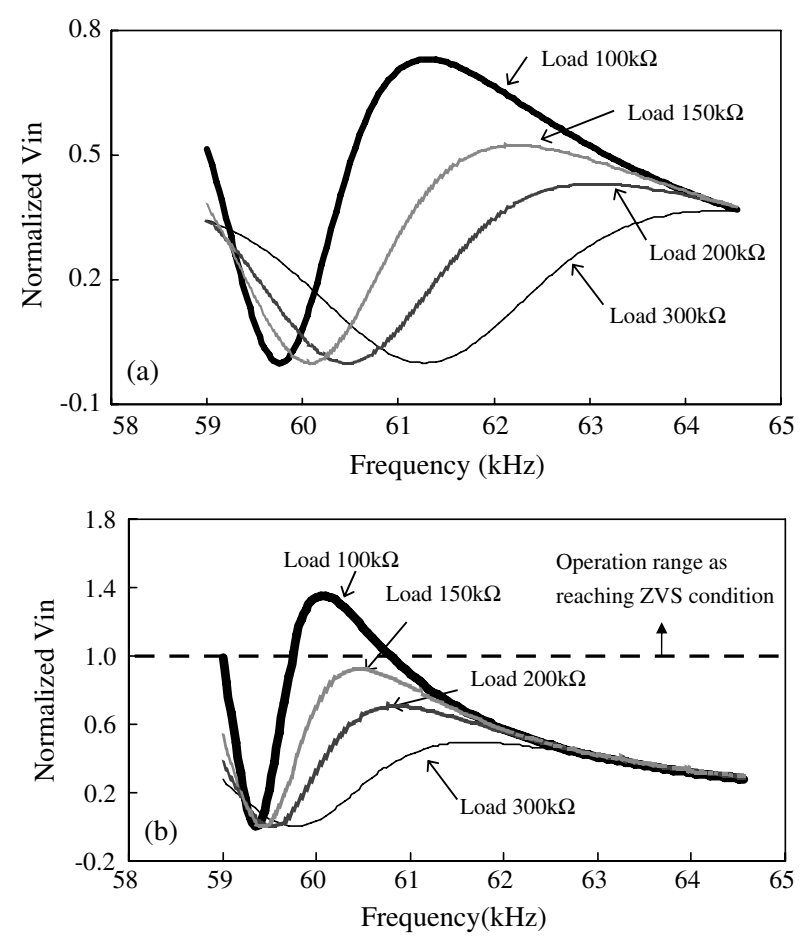

Figure 9. Charged voltage of (a) PTM2MM and (b) PTM1MM.

Assuming the dead time to be $1 / 5$ of the period, the normalized charged voltage of PTM1MM and PTM2MM can be drawn as in figure 9 by using equation (15). For comparison purposes, we defined a normalized $V_{\text {in }}=V_{C_{d 1}}(f) / V_{\mathrm{DC}}$ and used it to derive figure 9. In order to simulate the various operating conditions of a CCFL, a loading impedance of 100 , 150,200 and $300 \mathrm{k} \Omega$ were connected to the output of the piezoelectric transformer. Figure 9 shows that a lower loading impedance leads to a higher maximum charged voltage. Even when the loading impedance is as low as $100 \mathrm{k} \Omega$, the maximum charged voltage was only about 0.78 times of $V_{\mathrm{DC}}$ (figure 9(a)). That is, the PTM2MM will face difficulty in obtaining a ZVS condition when used to light a CCFL. To raise the charged/discharged voltage so as to obtain a ZVS condition, PTM1MM was fabricated to replace the PTM2MM. The charged voltage for the different loading impedances are shown in figure 9(b). As the thickness of the piezoelectric transformer changed from $2 \mathrm{~mm}$ to $1 \mathrm{~mm}$, the current to and from the internal inductance increased four times under the same input voltage, which helps to increase the changed voltage. More specifically, the maximum charged voltage of PTM1MM is more than 1.2 times of $V_{\mathrm{DC}}$ (figure 9 (b)) when the loading impedance was $100 \mathrm{k} \Omega$. This data clearly indicates that the MOSFET can obtain a ZVS condition when PTM1MM is used to light a CCFL. More specifically, a PTM1MM-based piezoelectric transformer obtains a ZVS condition when the driving frequency is in the range of $60-61 \mathrm{kHz}$ and when the loading impedance is $100 \mathrm{k} \Omega$ (figure 9(b)).

\section{Optimal operating region}

It is clear from the analysis and the experimental set-up mentioned above that a MOSFET of a piezoelectric inverter 

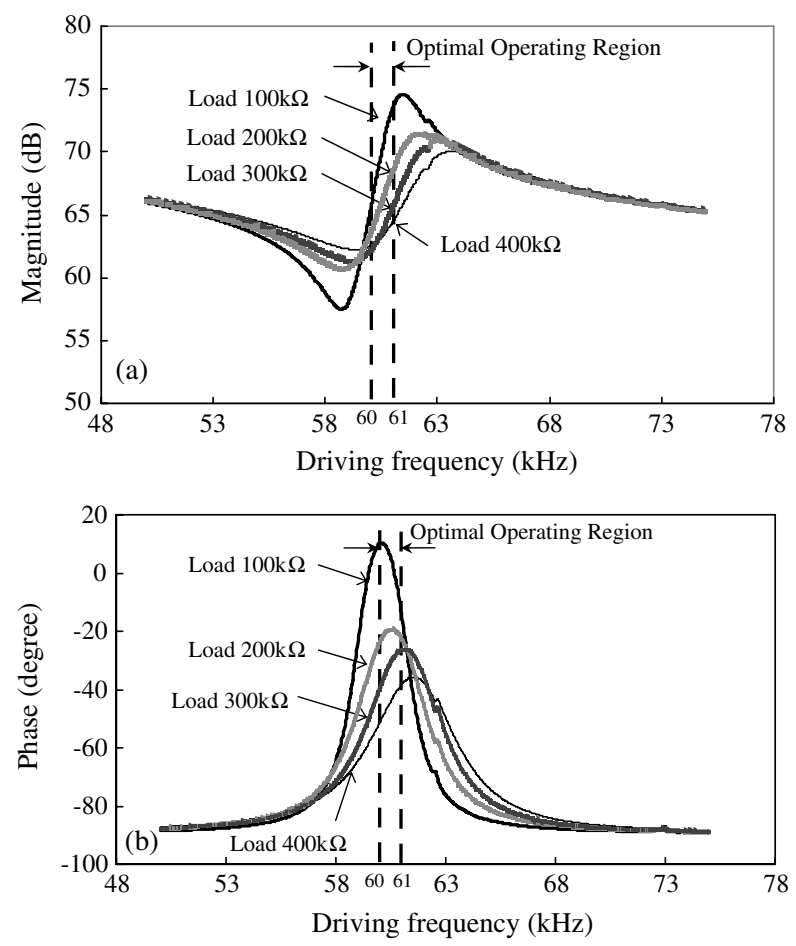

Figure 10. Magnitude (a) and phase (b) of a PTM1MM input impedance when the output section is connected to various loads which mimic a CCFL.

must obtain a ZVS condition in order to reach a maximum energy transfer efficiency. As the charge/discharge voltage will be changed whenever the driving frequency is changed, the driving frequency must be placed within the specific region if the ZVS condition is to be met. Traditionally, a piezoelectric transformer determines its optimal operating region by choosing the most important design parameters such as high step-up ratio, high energy transfer efficiency, etc. The measured input impedance of the PTM1MM is shown in figure 10. The loading impedance was changed from 100 to $400 \mathrm{k} \Omega$ in order to simulate the loading impedance of a CCFL. These experimental results showed that the frequency of maximum energy transfer efficiency is located between the resonant frequency and the anti-resonant frequency $[18,19]$. This maximum energy transfer frequency is very close to the frequency with a maximum phase degree as shown in figure 10(b). It is clear from figure 10(b) that the maximum phase angle is located between 59 and $61 \mathrm{kHz}$. The discontinuous peak shown in figures 10(a) and (b) can be attributed to the solder point on the surface of the piezoelectric transformer since we can change the peak jump by changing the solder point. Comparing figures 9(b), 10(a) and (b), we can see that the two different regions are very close to each other, which means that the PTM1MM can be used to light a CCFL by adopting a soft switching technology successfully as shown in equation (11). The data shown in figures 3-5 indicated that the PTM1MM can provide a full modal filtering effect, i.e. a trapezoidal input voltage can be used to drive the piezoelectric transformer directly. By using the design thinking mentioned above, a piezoelectric inverter which uses a soft switching technology to drive piezoelectric transformers
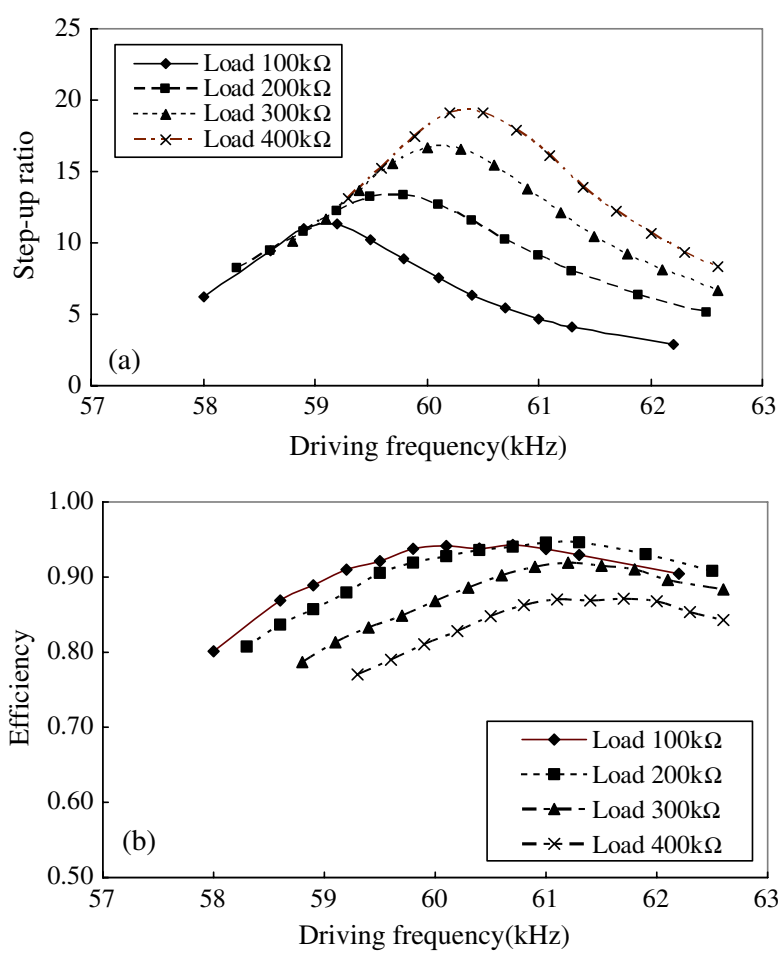

Figure 11. Step-up ratio (a) and efficiency (b) of a $1 \mathrm{~mm}$ Rosen type piezoelectric transformer with a quasi-modal electrode.

PTM1MM for LCD backlight applications was developed herein.

\section{High-power piezoelectric transformer}

Some experiments were done to examine the high-power property of the PTM1MM. A constant sinusoidal input voltage with different frequencies produced by function generator HP 3314 and power amplifier NF4052 were used to drive the piezoelectric transformer PTM1MM. The experimental results are shown in figure 11. A Tektronix high voltage probe was used to measure the input and output voltage waveforms. The input current was measured by using a Tektronix current probe A6302 and current probe amplifier AM503B. The output current was measured by using a small resistance connected in a series with loading impedance and the voltage across it to calculate the output current. Comparing the data shown in figure 11, it is clear that the maximum energy transfer efficiency frequency was slightly higher than the maximum step-up ratio frequency. The region of the two frequencies is called the optimal working frequency region traditionally, which is between 59.5 and $61 \mathrm{kHz}$ for this newly developed sample [19]. The maximum energy transfer efficiency for different loading impedances can be seen in figure 11 which shows that the maximum energy transfer will be higher than $90 \%$ when the loading impedance is changed from 100 to $300 \mathrm{k} \Omega$.

In figure 11 we can see that the step-up ratio of PTM1MM will be higher than 10 and the energy transfer efficiency will be greater than $90 \%$ in the optimal operating region. In order to examine the efficiency of PTM1MM in the region mentioned 
A modal actuator-based single-layer piezoelectric transformer for coilless soft switching inverters

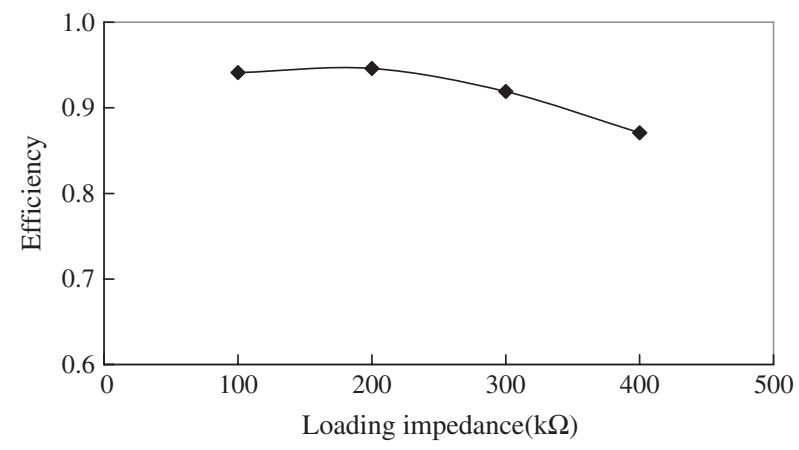

Figure 12. Maximum energy transfer efficiency for different loading impedances.
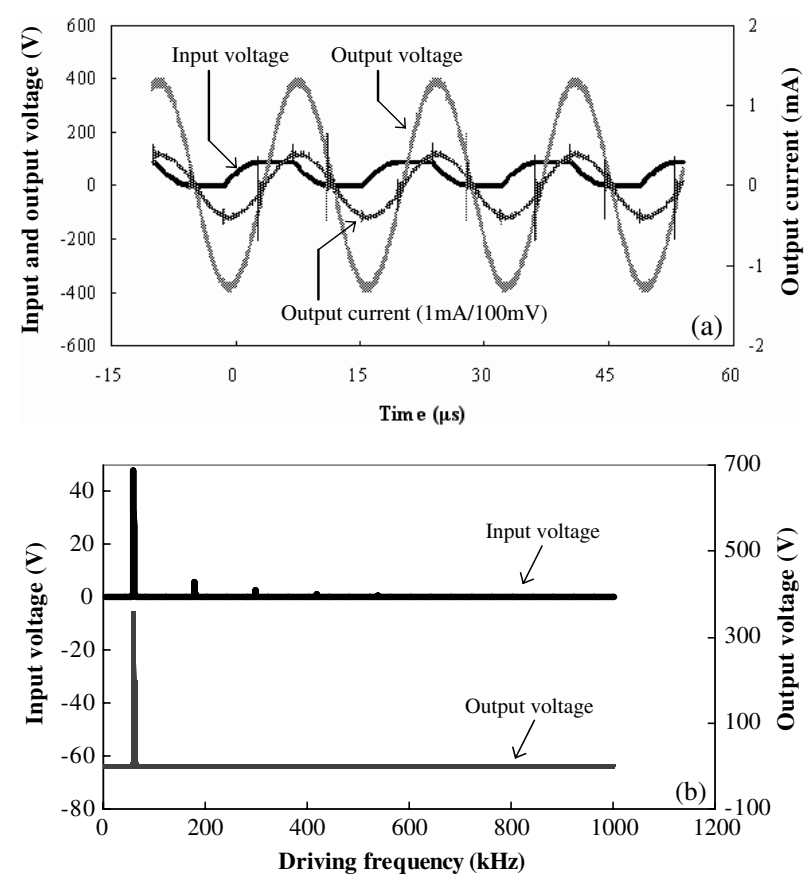

Figure 13. Input voltage, output voltage and output current (a) and FFT (b) of the input and output voltage when implementing the newly developed piezoelectric transformer.

above, another experiment was undertaken. An IC IR21844 and two N-type MOSFET IRF540 were used to form a highvoltage half-bridge driver and where the driving frequency was controlled by a function generator HP3314. The IC IR21844 possessed the capability to adjust the dead time by connecting a variable resistance externally. The output section of the PTM1MM was connected to a $100 \mathrm{k} \Omega$ loading impedance. The DC voltage was supplied from the Agilent DC Power Supply 6614C and the input power was measured from the built-in digital meter on the instrument. Using figures 9(b) and 11, we can see that the driving frequency chosen was $59.8 \mathrm{kHz}$, which is located within the optimal operating region. The input DC voltage and the input current were measured to be $90 \mathrm{~V}$ and $9.1 \mathrm{~mA}$. The input power was calculated by using the electrical signal obtained to be $0.819 \mathrm{~W}$. The output power was $0.766 \mathrm{~W}$, which was measured by using a high-speed LeCory LT364 oscilloscope. The waveform obtained can be seen in figure 13(a). By using the input and output power,

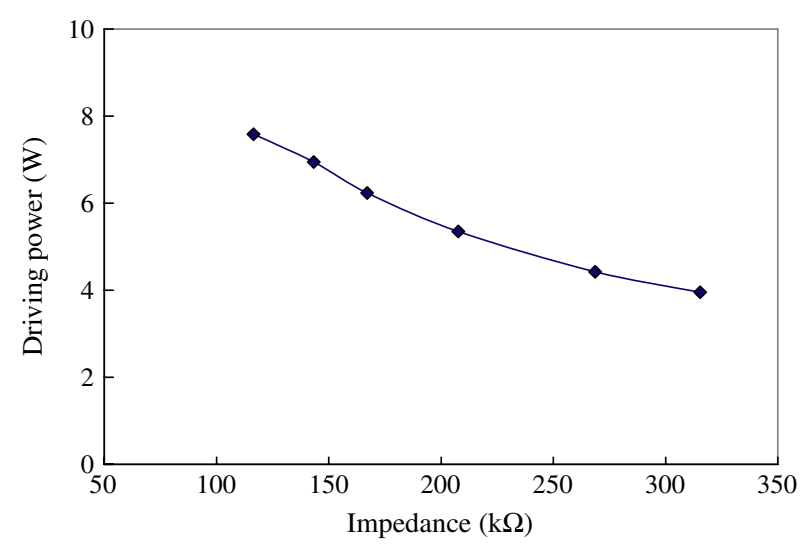

Figure 14. Properties of a $650 \mathrm{~mm}$ long CCFL.

the energy transfer efficiency was calculated to be $93.5 \%$. It is clear from figure 13(a) that the input voltage became a trapezoidal type, i.e. the two MOSFETs met the ZVS condition which agreed well with the theoretical derivations (figure 9(b)). The experimental results showed that total energy transfer efficiency (switch loss and piezoelectric transformer loss) were very close to the experimental results shown in figure 11(b). In other words, the energy loss of the two MOSFETs is almost zero when the driving circuit meets the ZVS condition. It also means that the quasi-modal electrode can help to retain an energy transfer efficiency even if a trapezoidal input voltage is used to drive the piezoelectric transformer.

To verify the modal filtering effect of the quasi-modal electrode, the measured input and output voltage were converted to spectra (figure 13(b)) by using a built-in fast Fourier transform function in a high-speed LeCory LT364 oscilloscope. It should be clear from figure 13(b) that the highfrequency voltage of the input voltage was filtered out as only the voltage with the first resonant frequency was transferred by the quasi-modal piezoelectric transformer. As a $650 \mathrm{~mm}$ long CCFL for LCD TV backlight was the potential load application, the optimal operating region and the equivalent CCFL impedance were measured (figure 14). From figure 14 we can see that an equivalent impedance of a CCFL will be in the range of 100-200 $\mathrm{k} \Omega$, which corresponds to the different tube currents used for brightness adjustment. It should be noted that an equivalent impedance of a CCFL is an important parameter in the design of a piezoelectric transformer as the equivalent impedance of the CCFL may influence the step-up ratio, energy transfer efficiency of the piezoelectric transformer and soft switching condition of the MOSFETs.

By combining the experimental results mentioned above, it is clear that if we are to determine the optimal operating region for a PTM1MM, parameters such as a step-up ratio, energy transfer efficiency and soft switching condition must be examined. From figures 9(b), 11(a) and (b), we can see that, if the driving frequency is in the region from 59.5 to $61 \mathrm{kHz}$, the piezoelectric transformer will possess a high energy transfer efficiency, high step-up ratio and meet a soft switching condition. The data shown in figures 12 and 14 shows that PTM1MM can be used to light a $650 \mathrm{~mm}$ long CCFL as its impedance region is very close to the CCFL impedance. 


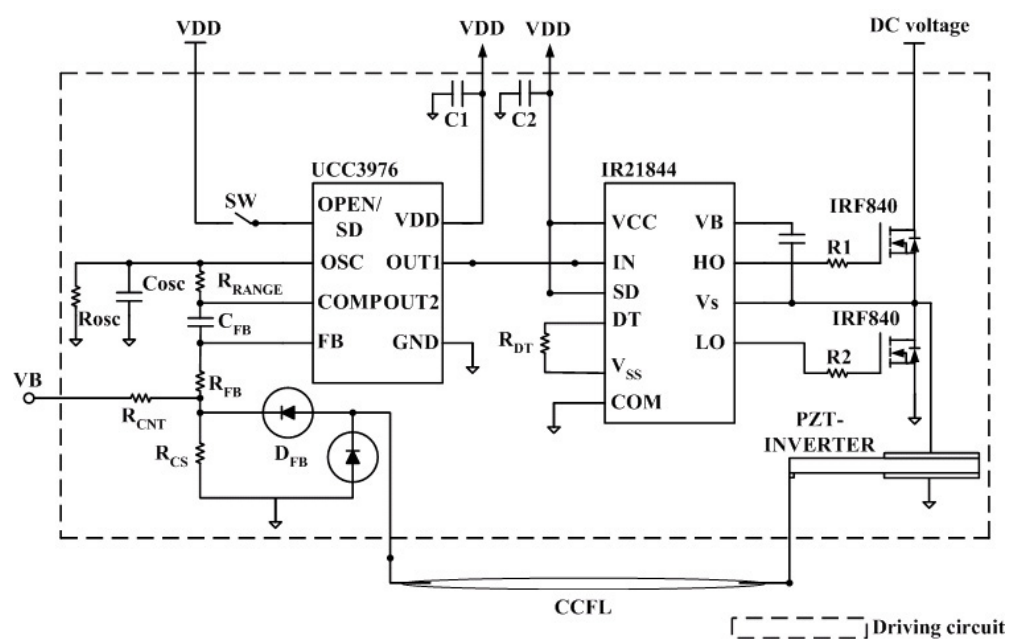

Figure 15. Driving circuit with soft switching technology.

\section{Coilless piezoelectric-based inverter with soft switching technology}

Based on the experimental results shown above, we can find that the PTM1MM can be used to construct an LCD TV backlight inverter. By adopting a soft switching technology to drive this newly developed piezoelectric transformer, no magnetic component is required. The inverter driving circuit can be seen in figure 15 which was designed by using two different integrated circuits (IC). The first IC was a Texas Instruments UCC3976 IC that was used to control the frequency of the inverter. The UCC3976 IC could sweep the frequency from high to low in order to ignite the CCFL properly. After lighting a CCFL a small resistance $R_{\mathrm{CS}}$ was used to measure the tube current. The driving frequency was adjusted to make the tube current constant. This is the constant current feedback control algorithm adopted traditionally and the main purpose of adopting this control here was to make sure the piezoelectric transformer worked well within the optimal operating region and make the CCFL maintain the same brightness.

The second IC was an International Rectifier IR21844. The IC IR21844 and the two N-type MOSFET IRF530s were used to form a half-bridge driver that could support an extrahigh DC voltage. In addition, the IR21844 IC possessed a lead denoted as DT, which was used to adjust the desired dead time by connecting a variable resistance $R_{\mathrm{DT}}$ to it. The maximum adjusted region of the dead time was $5 \mu$ s and the resonance frequency of the piezoelectric transformer used in this paper was about $60 \mathrm{kHz}$. Therefore, the maximum adjusted dead time region was about $1 / 3$ times the period.

As a single-layer piezoelectric transformer was used to light a CCFL for an LCD TV backlight herein, we needed to supply an extra-high DC voltage to the inverter. In practical applications, a high DC voltage can be provided from a PFC circuit (power factor correcting circuit). Similarly, a boost switching circuit can be used. The experimental results obtained by using this newly developed driving circuit to light a $650 \mathrm{~mm}$ long CCFL can be seen in figure 16. In figure 16, it is clear that the input voltage met a ZVS condition and no temperature rise was found on the two N-type MOSFETs.

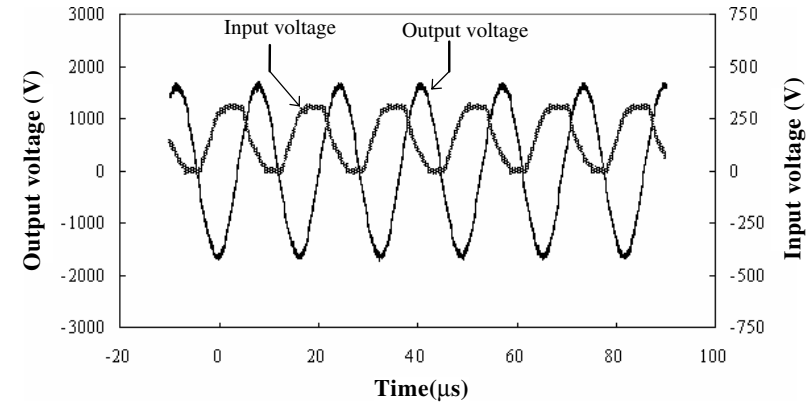

Figure 16. Input and output voltage of a piezoelectric transformer PTM1MM.

The controlled driving frequency was about $60.3 \mathrm{kHz}$ and the tube current was controlled to be constant. The data also showed that the input voltage changed from a rectified shape to a trapezoidal type, which helped to suppress the high frequency component embedded within the input voltage. We can see that the PTM1MM can be controlled to work under the optimal working frequency region and where the total efficiency (switching efficiency and piezoelectric transformer efficiency) can reach $86 \%$. The input power obtained was $6.48 \mathrm{~W}$ and the output power was $5.61 \mathrm{~W}$. A $650 \mathrm{~mm}$ long CCFL was ignited for more than $1 \mathrm{~h}$ and the temperature rise of the piezoelectric transformer PTM1MM was found to be about $10^{\circ}$.

\section{Conclusions}

The design thinking of a modal actuator integrated with a soft switching technology was combined to develop a piezoelectricbased inverter. A quasi-modal electrode was found to provide a full modal filtering effect to a trapezoidal input voltage waveform, which could improve the energy transfer efficiency of the piezoelectric transformer. The experimental results demonstrate that the total energy transfer efficiency (including switch and piezoelectric transformer) can reach $93.5 \%$ when the input power is $0.819 \mathrm{~W}$. When the loading impedance is 
A modal actuator-based single-layer piezoelectric transformer for coilless soft switching inverters

Table 2. Comparison between a quasi-modal PT and traditional PT under a ZVS condition.

\begin{tabular}{llll}
\hline & Quasi-modal PT + ZVS & Traditional PT + ZVS [7] & Traditional PT without ZVS [7] \\
\hline Size & $53 \mathrm{~mm} \times 7.5 \mathrm{~mm} \times 1 \mathrm{~mm}$ & $48 \mathrm{~mm} \times 8 \mathrm{~mm} \times 2 \mathrm{~mm}$ & - \\
$P_{\text {in }}$ & $819 \mathrm{~mW}$ & $376 \mathrm{~mW}$ & - \\
$P_{\text {out }}$ & $766 \mathrm{~mW}$ & $313 \mathrm{~mW}$ & - \\
$\eta$ & $93.5 \%$ & $83 \%$ & Less than $70 \%$ \\
\hline
\end{tabular}

$100 \mathrm{k} \Omega$, the PTM1MM can meet a ZVS condition theoretically. The soft switching technology was adopted to design a piezoelectric inverter without using any magnetic components. In table 2, a comparison between a quasi-modal PT and traditional PT under a ZVS condition can be seen. In general, traditional Rosen-type piezoelectric transformers suffer from energy leakage to a high resonance vibration mode. Both the experimental and the theoretical results show that a modal electrode implemented by using a quasi-modal electrode can eliminate the problem.

Finally, the proposed coilless piezoelectric-based inverter was completed. A $650 \mathrm{~mm}$ long CCFL was ignited. The total energy transfer efficiency reached $86.5 \%$ at an input power of $6.48 \mathrm{~W}$ and the temperature rise was found to be less than $10^{\circ} \mathrm{C}$.

\section{Acknowledgments}

The present study was financially supported by the National Science Council of Taiwan, through NSC grant 95-2622-E002-003. The authors would also like to thank ELECERAM Technology Co. Ltd. for continuously providing them with the PT as well as some funding for the research work. Thanks also go to Texas Instruments (USA) for generously providing the authors with the control ICs. The continuous financial support from AdvanceWave Technologies, Inc. as well as the Materials Research Laboratory, Industrial Technology Research Institute (ITRI) is also greatly appreciated. In addition, the authors would like to acknowledge the many helpful discussions with Mr Yuan-Ping Liu.

\section{References}

[1] Rosen C A Piezoelectric transformer US Patent Specification 2830274

[2] Yamamoto M, Member R, Shimada Y, Sasaki Y, Inoue T, Nakamura K and Ueha S 2002 A multilayered piezoelectric transformer operating in the third order longitudinal mode and its application for an inverter IEICE Trans. Electron. E85-C 1824-32

[3] Lin R L 2001 Piezoelectric transformer characterization and application of electronic ballast PhD Dissertation Department of Electrical Engineering, Virginia Polytechnic Institute
[4] Baker E M 2002 Design of radial mode piezoelectric transformers for lamp ballast applications MS Thesis Virginia Tech, Blacksburg

[5] Choi S J, Lee K C and Cho B H 2005 Design of fluorescent lamp ballast with PFC using a power piezoelectric transformer IEEE Trans. Ind. Electron. 52 1573-81

[6] Baker E M, Huang W, Chen D Y and Lee F C 2005 Radial mode piezoelectric transformer design for fluorescent lamp ballast application IEEE Trans. Power Electron. 20 1213-20

[7] Flynn A M and Sanders S R 2002 Fundamental limits on energy transfer and circuit considerations for piezoelectric transformers IEEE Trans. Power Electron. 17 8-14

[8] Lee C K 1987 Piezoelectric laminates for torsional and bending modal control: theory and experiment $P h D$ Dissertation Cornell University, Ithaca, New York

[9] Lee C K 1990 Theory of laminated piezoelectric plates for the design of distributed sensors/actuators: part I. Governing equations and reciprocal relationships J. Acoust. Soc. Am. 87 1144-58

[10] Lee C K 1992 Piezoelectric laminates: theory and experiments for distributed sensors and actuators Intelligent Structural Systems (The Netherlands: Kluwer-Academic) pp 75-167

[11] Hsu Y S, Lee C K and Hsiao W H 2003 Optimizing piezoelectric transformer for maximum power transfer Smart Mater. Struct. 12 373-83

[12] Hsu Y S, Lee C K and Hsiao W H 2005 Electric and mechanical fully coupled theory and experimental verification of Rosen-type piezoelectric transformers IEEE Trans. Ultrason. Ferroelectr. Freq. Control 52 1829-39

[13] Huang Y T, Hsiao W H, Hsu Y S and Lee C K 2005 High-power backlight inverter for LCD-TV using piezoelectric transformers Proc. 15th Int. Conf. on Adaptive Structures and Technologies 2005

[14] Huang Y T, Wu W J and Lee C K 2007 High-power backlight for LCD-TVs using piezoelectric transformers J. Intell. Mater. Syst. Struct. 18 601-9

[15] Lee C K et al 1999 A high performance Doppler interferometer for advanced optical storage systems Japan. J. Appl. Phys. 38 1730-41

[16] Lin C Y 1997 Design and analysis of piezoelectric transformer converters PhD Dissertation Department of Electrical Engineering, Virginia Polytechnic Institute

[17] Katz H W 1959 Solid State Magnetic and Dielectric Devices (New York: Wiley) pp 94-126

[18] Sakurai K, Ohnishi K and Tomikawa Y 1999 Presentation of a new equivalent circuit of a piezoelectric transformer under high-power operation Japan. J. Appl. Phys. 38 5592-7

[19] Shimamura J, Sakamoto M and Kamitani K 2002 Method and apparatus for driving piezoelectric transformer US Patent Specification 6348755 